\title{
La toma de decisión en el fútbol: una perspectiva desde la integración en el entrenamiento específico del deporte
}

\author{
Decision-making in soccer: \\ a perspective from integration in specific sports training \\ *,** Luis Alberto Benavides Roca, *,** Pía Constanza Santos Vásquez, \\ *** Gastón Díaz Coria, **** María Isabel Benavides Roca
}

Benavides, L., Santos, P., Díaz, G. \& Benavides, I. (2018). La toma de decisión en el fútbol: una perspectiva desde la integración en el entrenamiento específico del deporte. Revista Ciencias de la Actividad Física UCM, $\mathrm{N}^{\circ}$ 19(1) enero-junio, 1-10. DOI: http://doi.org/10.29035/rcaf.19.1.6

\section{RESUMEN}

El fútbol es uno de los deportes que presenta mayor interacción, pues existen múltiples factores que intervienen en el desarrollo de esta disciplina, es por ello que se hace fundamental analizar la toma de decisiones de los futbolistas al momento de verse sometido a situaciones de gran estrés. El objetivo de la presente revisión es analizar la literatura a partir de la toma de decisiones en el fútbol estableciendo directrices de entrenamiento que involucren actividades con toma de decisiones. Se obtuvieron artículos a partir de las bases de datos, Scielo, Lilacs, Ebsco y Web of Sciene. Se obtuvo 14 artículos que respondían de mejor forma al objetivo planteado. Finalmente se señala que la toma de decisión debe ser integrada a los modelos de entrenamiento para conseguir el máximo desempeño del futbolista y del equipo.

\section{PALABRAS CLAVE}

Fútbol, toma de decisión, deporte y entrenamiento.

\begin{abstract}
Soccer is one of the sports that presents the most interactions, since there are multiple factors involved in the development of this discipline, which is why it is essential to analyze the players' decisionmaking at the moment when they are subjected to high stress situations. The objective of the present review is to analyze the literature based on decision-making in soccer by establishing training guidelines that involve activities with decision-making. Articles were obtained from the following databases; Scielo, Lilacs, Ebsco and Web of Science. The 14 articles that were obtained responded better to the proposed objective. Finally, it is pointed out that decision-making must be integrated into the training models to achieve the maximum performance of the soccer players and the team.
\end{abstract}

Key words

Soccer, Decision making, Sports and Training. 


\section{INTRODUCCIÓN}

El fútbol resulta ser uno de los deportes más populares y más practicado en el mundo, muestra de ello, son millones de personas que día a día, participan de este deporte (Boniface, 2006). Por tanto, la importancia que tiene la investigación en esta disciplina es muy relevante, ya que conlleva desde lo social a lo competitivo, siendo esto último lo que se intenta analizar, para entender los componentes que influyen en el futbolista (Reina-Gómez y Hernández-Mendo, 2002).

Dentro de las dinámicas propias del fútbol, están los entrenamientos que se llevan a cabo día a día, buscando el máximo desempeño al jugador. Se estructuran modelos de planificación donde existe una parte específica, que conlleva aspectos propios del deporte, incluyendo acciones transferibles a la competición, sin embargo, siempre está asociado a modelos analíticos pocos dinámicos (Gomes Tubino, 1997).

Las características propias del deporte, hacen relacionar las acciones con un aspecto cognitivo del deportista, donde se intenta conseguir la máxima información del entorno para responder de la mejor forma ante una necesidad de movimiento (Antunez-Medina, 2004). Estas acciones permiten que los jugadores exploren y descubran nuevas fuentes de información, ante las distintas interacciones que se crean en el juego (Gréhaigne, Bouthier \& David, 1997).

A partir de lo anterior, el fútbol como competencia, es una de las disciplinas que genera un gran estrés cognitivo para los jugadores, pues el número de factores que intervienen para una respuesta motriz son muy variados (compañeros, contrincantes, balón, árbitros, césped, hinchada, etc). El tiempo de respuesta debe ser rápido y preciso con un alto nivel de pericia, sin embargo, entre más rápido responda, las opciones de equivocarse son mayores (Iglesias, et al 2003). El manejo de esta dinámica de juego y la comprensión de las competencias específicas del fútbol (táctica) llevarán a conseguir un jugador experto (Ruiz, et al 2014). Por tanto, la toma de decisiones resulta fundamental a la hora de realizar este deporte, pues jugar bien, consiste en elegir la opción de juego más adecuada en el momento justo o exacto y ser capaz de llevarla a cabo, siendo fundamental el manejo temporal y la exigencia de precisión de la acción (García, Ruiz, Graupera, 2009).

La comprensión de los procesos subyacentes de un deportista estará reflejado en la capacidad de decisión que posea, es decir adaptar y regular sus movimientos en función de las posibilidades de acción (Duarte et al, 2010).

Estos procesos decisionales están constituidos por tres fases. La primera de preparación de la decisión, que se centra en el análisis de la situación y de la posibilidad de acción; la segunda, acto de decisión, donde se escogerá el gesto técnico-táctico a realizar; y la tercera denominada de realización y control de la decisión, incluye los ajustes de la acción escogida y una valoración de la decisión tomada (Kozang, 1992; Iglesias, et al, 2002).

Bajo la perspectiva del aprendizaje, existen dos corrientes científicas que estudian la toma de decisiones en los deportes. La primera busca identificar las diferencias entre jugadores expertos y novatos (Griffin et al., 2001), mientras que la segunda se refiere al dominio en específico con que los deportistas asisten a los entrenamientos (MacPhail, Kirk, \& Griffin, 2008).

A partir de esta evidencia, es posible creer que la toma de decisiones en el deporte se puede entrenar y conseguir un mayor desempeño cuando se somete a un sujeto a estímulos que desarrollen aspectos de la cognición. Ruiz \& Graupera (2005) presentan un modelo que centra su análisis en los aspectos emocionales y motivacionales del deportista a la hora de tomar decisiones en contextos de entrenamiento y competición, por su parte Iglesias et al (2005), propone un programa de supervisión reflexiva, que busca generar una retroalimentación al sujeto tras una acción correcta e incorrecta. No obstante, los trabajos señalados no son incluidos dentro de las actividades mismas 
del entrenamiento, es por ello que el presente trabajo tiene como objetivo analizar la literatura a partir de la toma de decisiones en el fútbol estableciendo modelos de entrenamiento que lleven a cabo actividades con toma de decisiones.

\section{METODOLOGÍA}

En el contexto de una revisión bibliografía, se plantea las siguientes interrogantes: ¿Qué indicadores de la toma de decisión podrían ser desarrollados en el ámbito del fútbol?, ¿Qué usos y aplicaciones se podrían considerar en el entrenamiento del fútbol?

La estrategia de búsqueda bibliográfica efectuada ha sido localizar artículos en las bases de datos informatizadas on-Line más importantes del área del entrenamiento deportivo y neurociencia, incluyendo Scielo, Ebsco Host, Lilacs y web of science, publicados entre los años 2010 y 2017. Se utilizaron como palabras clave: toma de decisión y fútbol (decisión making and football; Tomada de decisão e futebol) expresadas en idioma inglés, español y portugués.

Se consideró como criterios de inclusión: estudios de revisión, descriptivos e intervención, siendo excluidos: estudios científicos en forma de resumen, comunicaciones y/o cartas.

La secuencia de búsqueda consistió en primera instancia realizar una indagación abierta donde se discriminaba por el título del estudio, en segunda, instancias se pasaba a leer el resumen y se veía la pertinencia con el objetivo de la presente revisión y en tercera instancia, se leía por completo el artículo, buscando responder a las preguntas planteadas anteriormente. 


\section{RESULTADOS}

\section{Tabla 1}

Artículos seleccionados.

\begin{tabular}{|c|c|c|c|}
\hline Autor & Año & Artículo & Base de datos \\
\hline Roca y Williams & 2017 & $\begin{array}{l}\text { Does decision making transfer across similar and } \\
\text { dissimilar sports? }\end{array}$ & Scielo \\
\hline Romero et al., & 2016 & $\begin{array}{l}\text { Constrangimentos espácio-temporais sobre a } \\
\text { tomada de decisão do tipo de remate na grande } \\
\text { área do futebol }\end{array}$ & Web of science \\
\hline Gonzaga et al., & 2014 & $\begin{array}{l}\text { Affective Decision-Making and Tactical Behavior } \\
\text { of Under-15 Soccer Players }\end{array}$ & Ebsco \\
\hline Roca, Williams y Ford & 2012 & $\begin{array}{l}\text { Developmental activities and the acquisition of } \\
\text { superior anticipation and decision making in } \\
\text { soccer players }\end{array}$ & Lilacs \\
\hline González-Víllora et al., & 2015 & $\begin{array}{l}\text { Evolución de la toma de decisiones y la habilidad } \\
\text { técnica en fútbol }\end{array}$ & Lilacs \\
\hline Buszard, Farrow \& Kemp & 2012 & $\begin{array}{l}\text { Examining the influence of acute instructional } \\
\text { approaches on the decision-making performance } \\
\text { of experienced team field sport players }\end{array}$ & Lilacs \\
\hline Ruiz-Perez et al., & 2015 & $\begin{array}{l}\text { Autopercepción de inteligencia contextual para } \\
\text { jugar y de decisional en el fútbol competencia }\end{array}$ & Ebsco \\
\hline González-Víllora et al., & 2011 & $\begin{array}{l}\text { Conocimiento táctico y toma de decisiones en } \\
\text { jóvenes jugadores de fútbol ( } 10 \text { años })\end{array}$ & Ebsco \\
\hline Woods et al., & 2015 & $\begin{array}{l}\text { Discriminating talent-identified junior Australian } \\
\text { football players using a video decision-making } \\
\text { task }\end{array}$ & Ebsco \\
\hline Frybort et al., & 2016 & $\begin{array}{l}\text { Does Physical Loading Affect The Speed and } \\
\text { Accuracy of Tactical Decision-Making in Elite } \\
\text { Junior Soccer Players? }\end{array}$ & Ebsco \\
\hline Smith et al., & 2016 & $\begin{array}{l}\text { Mental fatigue impairs soccer-specific decision- } \\
\text { making skill }\end{array}$ & Ebsco \\
\hline Praxedes-Pizarrol et al., & 2017 & $\begin{array}{l}\text { The effects of a comprehensive teaching program } \\
\text { on execution skills of young footballers dribbling } \\
\text { and passing decision-making and }\end{array}$ & Ebsco \\
\hline Hastie & 2012 & $\begin{array}{l}\text { The impact of a hybrid Sport Education-Invasion } \\
\text { Games Competence Model soccer unit on students' } \\
\text { decision making, skill execution and overall game } \\
\text { performance }\end{array}$ & Ebsco \\
\hline Otero-Saborido et al., & 2012 & $\begin{array}{l}\text { Validación de instrumentos para la medición del } \\
\text { conocimiento declarativo y procedimental y la } \\
\text { toma de decisiones en el fútbol escolar }\end{array}$ & Ebsco \\
\hline
\end{tabular}




\section{DISCUSIÓN}

\section{Toma de decisiones en el fútbol}

La toma de decisión dentro de los deportes colectivos resulta ser considerada como un proceso funcional y emergente, en que la selección se hace convergente con el objetivo deseado (Araujo, Davids, \& Hristovski, 2006). Mesquita, Farias \& Hastie (2012), determinó los efectos de la enseñanza de modelos de competencias por sobre las decisiones deportivas y las habilidades tácticas, obteniendo una mejora en estos componentes, tras la integración del modelo cognitivo para los niños.

En la literatura es posible encontrar diversos estudios que encausan la toma de decisión dentro del deporte, generalmente identificándola como un agente mediador entre el alto rendimiento y la acción correcta, de ahí que actualmente resulta ser un objeto de estudio. A partir de esto Woods et al., (2015) establece un paralelo entre el talento y el correcta decisión técnica y táctica en niños australianos, también González-Villora, et al (2015), realiza una investigación en niños de 10 años, donde evalúa la toma de decisión en el fútbol a través de las acciones propia de los jugadores, considerándose exitosas y no exitosas de acuerdo a la decisión que ellos tomaran durante el encuentro, basándose en componentes técnicos de pase, control, regate, conducción, tiro, fijar y desmarque, lo que involucra una conexión existente entre las respuestas motrices y los procesos mentales asociados a aspectos tácticos del propio deporte, lo cual también fue estudiado por Otero-Saborido et al., (2012), en situaciones más específicas de 2 vs 1 en el fútbol.

Por su parte, González-Víllora, García-López, \& Contreras-Jordán (2011) trabajaron en paralelo con la ejecución motriz y la toma de decisiones, encontrando correlaciones directamente significativas entre estas dos variables, lo que hizo identificar como los procesos cognitivos desarrollados en los sujetos eran capaces de ser reflejados en un acto motor.

En el fútbol, los jugadores se encuentran inmersos en un ambiente inestable en el que continuamente se necesita tomar decisiones para adaptarse a las situaciones que se dan, por ello se debe escoger la opción de juego más correcta y establecer el momento más apropiado para llevarlo a cabo (Thomas \& Thomas, 1994). A partir de esto, se hace importante en la dinámica del deporte, pues en el estudio de Gonzaga et al., (2014) se señala que la toma de decisiones afectiva puede influir en el comportamiento táctico de los jugadores de fútbol menores de 15 años, ya que es una función ejecutiva relacionada con el análisis de costobeneficio en situaciones donde las ganancias y pérdidas implican consecuencias directas para el sujeto. El artículo anteriormente señalado resulta interesante, pues se observa la toma de decisión con el componente de la neurociencia y desde la perspectiva emocional, lo que hace explicar que los procesos psicológicos no actúan de forma independiente, y que el futbolista posee una dimensión emocional y tácticacognitiva (Ruiz \& Arruza, 2005).

La toma de decisión, está relacionada con las propias capacidades del deportista, con la tarea que debe resolver y con las características del entorno de actuación.

\section{AMBIENTE}

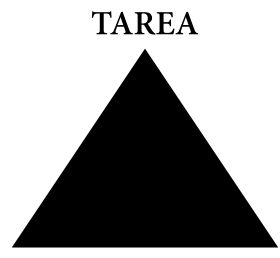

\section{DEPORTISTA}

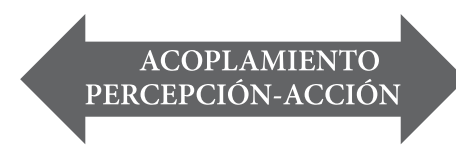

Figura 1. Factores que influyen en la decisión (adaptado de Araujo, 2006).

La Figura 1 refleja los factores que intervienen a la hora de cumplir una tarea. Como se puede ver, en la base se encuentran lo que es el ambiente, que se interpreta como las influencias que se tienen del exterior, generalmente asociado a donde se desarrolla la tarea y con 
quién, por su parte el deportista, hace referencia a las características propias del sujeto que actúa, como lo antropométrico o lo psicológico. Cuando se logran conjugar ambos aspectos se establece un patrón de coordinación funcional, el cual puede ser mejorado con la experiencia, dado por la entrenabilidad del sujeto. El nivel de reciprocidad y continuidad entre la información y el movimiento, es una propuesta metodológica esencial en la concepción de las tareas experimentales, ya que implica la posibilidad de movimiento y la decisión hacia la tarea que se deba cumplir (Davids, Button, Araújo, Renshaw \& Hristovski, 2006). En este sentido la toma de decisión resulta ser un proceso donde interfieren las limitaciones del individuo y el desarrollo de la tarea (Araújo \& Travassos, 2009).

En el estudio de Ruiz-Perez et al., (2015) se aprecia que los jugadores de fútbol que tienen mayor nivel en el deporte, presentan un mayor desempeño en la toma de decisiones y tienen una mayor autopercepción conceptual, lo que indica que estos factores permiten una ejecución más elevada de la tarea. Similar a lo propuesto por Buszard, Farrow \& Kemp, (2012) los cuáles estudiaron a jugadores de fútbol de distintos niveles, concluyendo que los sujetos con menor experiencia se ven afectados por las instrucciones que se dan en el campo de juego, llevándolos a cometer más errores en las tareas específicas al deporte tanto en lo táctico como en lo técnico. Por tanto, es posible identificar que los jugadores con un alto nivel deportivo son capaces de percibir mejor su entorno y a partir de eso, generar una decisión correcta, no obstante, las características propias del fútbol demandan al jugador un estrés cognitivo que repercute en la calidad de la toma de decisiones (Smith, 2016).

Roca, Wiliams \& Ford, (2012), establecieron que las capacidades decisionales eran duraderas en el tiempo y que a mayor cantidad de horas de entrenamiento las respuestas cognitivas y decisionales eran de mayor calidad. Resulta significativo lo expresado anteriormente pues, Roca y Williams (2017) proponen que la experiencia de un deportista lo llevará a responder de buena forma en diversas acciones de similar contexto, ya que evidenciaron que mujeres futbolistas respondían de buena forma no tan sólo a situaciones de fútbol, sino también en contextos de tenis y básquetbol.

\section{Implementación en el entrenamiento}

Existen modelos de enseñanza en los deportes desde una perspectiva comprensiva, donde se busca el conocimiento y desarrollo táctico por sobre el técnico, se comprende este pensamiento como un modelo contemporáneo donde la toma de decisiones toma relevancia para generar cambios en el desempeño del futbolista (Contreras, García-López y Cervelló, 2005). Turner, \& Martinek (1999), trabajaron en hockey basándose en concientización táctica, observando un desarrollo del desempeño de los jugadores en el ámbito decisional y estableciendo una vinculación de aspectos cognitivos en los entrenamientos.

Dentro del mundo del fútbol, los entrenamientos son efectuados para desarrollar modelos de juego y potenciar el aspecto técnico de los jugadores. Sin embargo, se dejan de lado aspectos asociados a la cognición y se da más realce a estructuras poco dinámicas que buscan la automatización del movimiento. Este precepto no involucra el concepto general de deportista, por lo que se hace interesante implementar dentro del entrenamiento modelos asociados a la toma de decisiones que involucre los procesos de la neurociencia.

Duarte et al. (2010), Propone que existe la relación entre la toma decisiones y la técnica en el fútbol, donde se aprecia que el desarrollo de estos componentes va a estar ligado de forma directa, específicamente analizaron el dribling en relación con la distancia interpersonal y la velocidad relativa entre los jugadores, en un contexto de 1 vs 1 . Junto con esto Headrick et al (2011), también trabajó la técnica a partir de la influencia de la distancia de la portería y la relación interpersonal entre los delanteros y defensa, obteniendo patrones decisionales de acuerdo a estos componentes. Por su parte Romero et al., (2016) analizó la decisión de la trayectoria del balón (en dirección hacia la portería), de acuerdo a las variables espaciotemporales, obteniendo, que la elección estaba 
influenciada por la superficie de contacto y la interacción entre los jugadores. Lo anterior establece que existen componentes de la toma de decisión que pueden ser involucradas dentro del entrenamiento técnico del fútbol.

Vickers (2007) señala que las habilidades decisionales se mejoran a través de un proceso de aprendizaje específico, aplicando modelos cognitivos en contextos variados. Específicamente se observa en la literatura intervenciones en la toma de decisión basadas en juegos cognitivos vinculados a acciones técnicas de pase y dribling, los cuales provocaban una mejor respuesta a situaciones de juego (Praxedes-Pizarro et al., 2017)

La capacidad de un jugador de fútbol para controlar y gestionar su comportamiento en una situación de juego refleja no solo la toma de decisiones táctica rápida y precisa, sino también la implementación inmediata de una tarea motora durante las condiciones de ejercicio intermitente. Frýbort, et al (2016), analizó la relación entre la intensidad de ejercicio y el tiempo de respuesta visual motora en una acción ofensiva de juego, obteniendo cambios no significativos en la precisión de la respuesta motora durante el ejercicio de alta intensidad, el ejercicio intermitente intenso, el ejercicio aeróbico intenso y la inactividad motora. Además, se encontró una relación significativa entre el tiempo de reacción y la precisión en la respuesta motora. Por lo que la toma de decisión correcta en situaciones de juego típicas representa más bien un mecanismo automático. Por tanto, involucrar el aspecto decisional de forma aislada al contexto del entrenamiento resulta inapropiado, es por ello es que se propone la integración de actividades que se asocien a componentes cognitivos acompañados de acciones específicas del fútbol, tanto técnica como táctica.

\section{CONCLUSIÓN}

Finalmente, se cree interesante la implementación de un modelo abierto donde el futbolista deba escoger algunas acciones técnicas y/o táctica, repercutiendo en alguna respuesta mayor de sus compañeros. Esto generará una mayor plasticidad cerebral y por ende un progreso del proceso cognitivo asociado a un desarrollo de la toma de decisión correcta.

Se sugiere mayor indagación en la integración de estos modelos en la etapa de iniciación del fútbol, pues es fundamental para el desarrollo cognitivo y motriz de niño, ya que los estímulos que se plantean son relacionados con la evolución deportiva de un sujeto. 


\section{REFERENCIAS BIBLIOGRÁFICAS}

Antúnez Medina, A., Ureña Villanueva, F., Velandrino Nicolás, A.P. \& García Parra, M.M. (2004). Valoración de la efectividad de interceptación com êxito de la portera de balonmano ante el lanzamiento trás la aplicación de um programa perceptivo-motor. Revista Internacional de Medicina y Ciencias de la Actividad Física y el Deporte, 4(15), 192-203.

Araujo, D. \& Travassos, B. (2009). Improving Decision Making Skill in Soccer. In B. Zoudji, Association des chercheurs francophones en football (Eds.). Science \& football: recherches et connaissances actuelles. (pp. 299-306). France: Presses universitaires de Valenciennes.

Araujo, D., Davids, K., \& Hristovski, R. (2006). The ecological dynamics of decision making in sport. Psychology of sport and exercise, 7(6), 653-676.

Boniface, P. (2006). El fútbol, fenómeno global por excelencia. Dossier La Vanguardia, 6-14.

Buszard, T., Farrow, D., \& Kemp, J. (2012). Examining the influence of acute instructional approaches on the decisionmaking performance of experienced team field sport players. Journal of sports sciences, 31(3), 238-247.

Contreras, O. R., García-López, L. M. \& Cervelló, E. (2005). Transfer of tactical knowledge: from invasion games to hockey. Journal of Human Movement Studies, 49, 193-213.

Davids, K., Button, C., Araújo, D., Renshaw, I. \& Hristovski, R. (2006). Movement models from sports provide representative task constraints for studying adaptive behavior in human motor systems. Adaptive Behavior, 14, 73-95.
Duarte, R., Freire, L., Gazimba, V., \& Araújo, D. (2010). A emergência da tomada de decisão no futebol: da decisão individual para a colectiva. In Nogueira, C. (Eds.), Psicologia Do Desporto: Actas do VII Simpósio Nacional de Investigação Em Psicologia. Braga: Universidade do Minho.

Frýbort, P., Kokštejn, J., Musálek, M., \& Süss, V. (2016). Does Physical Loading Affect The Speed and Accuracy of Tactical Decision-Making in Elite Junior Soccer Players?. Journal of sports science \& medicine, 15(2), 320-326.

García, V., Ruiz, LM., \& Graupera, JL. (2009). Perfiles decisionales de jugadores y jugadoras de voleibol de diferente nivel de pericia. Revista Internacional de Ciencias del Deporte, 14(5), 123-137.

Gomes Tubino, MJ. (1997). Metodología Científica Do Treinamento Desportivo. $8^{\circ} \mathrm{ed}$. Sao Paulo: IBRASA.

Gonzaga, AdS., Albuquerque, MR., MalloyDiniz, LF., Greco, PJ., \& Teoldo da Costa, I. (2014). Affective DecisionMaking and Tactical Behavior of Under-15 Soccer Players. PLoS ONE 9(6), e101231. https://doi.org/10.1371/journal.pone.0101231

González Víllora, S., García López, L. M., Pastor Vicedo, J. C., \& Contreras Jordán, O. R. (2011). Conocimiento táctico y la toma de decisiones en jóvenes jugadores de fútbol (10 años). Revista de Psicología del Deporte, 20(1), 79-97.

González-Víllora, S., García-López, L. M., \& Contreras-Jordán, O. R. (2015). Evolución de la toma de decisiones y la habilidad técnica en fútbol. Revista Internacional de Medicina y Ciencias de la Actividad Física y del Deporte/International Journal of Medicine and Science of Physical Activity and Sport, 15(59), 467-487. 
Gréhaigne, J. F., Bouthier, D. \& David, B. (1997). Dynamic-system analysis of opponent relationships in collective actions in soccer. Journal of Sports Sciences, 15, 137-149.

Griffin, L. L., Dodds, P., Placek, J. \& Tremino, F. (2001). Middle school estudents conceptions of soccer: Their solutions to tactical problems. Journal of Physical Education, 20(4), 324-340.

Headrick, J., Davids, K., Renshaw, I., Araújo, D., Passos, P., \& Fernandes, O. (2011). Proximity-to-goal as a constraint on patterns of behaviour in attacker-defender dyads in team games. Journal of Sports Sciences, 30(3), 247-253.

Iglesias, D., Sanz, D., García, T., Cervelló, E. M., \& Del Villar, F. (2005). Influencia de un programa de supervisión reflexiva sobre la toma de decisiones y la ejecución del pase en jóvenes jugadores de baloncesto. Revista de Psicología del Deporte, 14(2), 209-223.

Iglesias, D., Moreno, P., Ramos, L.A., Fuentes, J.P., Julián, J.A. \& Del Villar, F. (2002). Un modelo para el análisis de los procesos cognitivos implicados en la toma de decisiones en deportes colectivos. Revista de Entrenamiento Deportivo, 16(2), 9-14.

Iglesias, D., Ramos, L.A., Fuentes, J.P., Sanz, D. \& Del Villar, F. (2003). El conocimiento y la toma de decisiones en los deportes de equipo: una revisión desde la perspectiva cognitiva. Revista de Entrenamiento Deportivo 17(2), 5-11.

Konzag, I. (1992). Actividad cognitiva y formación del jugador. Revista de Entrenamiento Deportivo, 6(6), 35-44.

MacPhail, A., Kirk, D., \& Griffin, L. (2008). Throwing and catching as relational skills in game play: Situated learning in a modified game unit. Journal of Teaching in Physical Education, 27(1), 100115.
Mesquita, I., Farias, C., \& Hastie, P. (2012). The impact of a hybrid sport education-invasion games competence model soccer unit on students' decision making, skill execution and overall game performance. European Physical Education Review, 18(2), 205-219.

Otero Saborido, F. M., González Jurado, J. A., \& Calvo Lluch, Á. (2012). Validación de instrumentos para la medición del conocimiento declarativo y procedimental y la toma de decisiones en el fútbol escolar. Retos. Nuevas tendencias en Educación Física, Deporte y Recreación, 22, 65-69.

Praxedes-Pizarro, A., Moreno-Dominguez, A., Sevil-Serrano, J., Garcia-Gonzalez, L., \& Del Villar, F. (2017). The effects of a comprehensive teaching program on dribbling and passing decision-making and execution skills of young footballers. Kinesiology 49(1), 74-83.

Reina Gómez, A., \& Hernández Mendo, A. (2012). Revisión de indicadores de rendimiento en fútbol. Revista Iberoamericana Ciencias Actividad Física Deportiva, 1(1), 1-14.

Roca, A., \& Williams, A. M. (2017). Does decision making transfer across similar and dissimilar sports? Psychology of Sport and Exercise, 31, 40-43.

Roca, A., Williams, A. M., \& Ford, P. R. (2012). Developmental activities and the acquisition of superior anticipation and decision making in soccer players. Journal of sports sciences, 30(15), 16431652.

Romero Clavijo, F. A., Alvares Denardi, R., Travassos, B., \& Corrêa, U. C. (2016). Constrangimentos espácio-temporais sobre a tomada de decisão do tipo de remate na grande área do futebol. $\mathrm{Mo}$ tricidade, 12(2), 80-87. 
Ruiz, L.M. \& Arruza, J. (2005). El proceso de toma de decisiones en el deporte. Clave de la eficiencia y el rendimiento óptimo. Barcelona: Paidós.

Ruiz, L.M. \& Graupera, J.L. (2005). Dimensión subjetiva de la toma de decisiones en el deporte: Desarrollo y validación del cuestionario CETD de estilo de decisión en el deporte. Motricidad, 14, 95107.

Ruiz, L.M., García, V., Palomo, M., Navia, J.A., \& Miñano, J. (2014). Inteligencia contextual y pericia en el fútbol / Contextual intelligence and expertise in soccer. Revista Internacional de Medicina y Ciencias de la Actividad Física y el Deporte, 14(54), 307-317.

Ruiz-Pérez, L. M., Navia, J. A., Miñano-Espín, J., García-Coll, V., \& Palomo-Nieto, M. (2015). Autopercepción de inteligencia contextual para jugar y de competencia decisional en el fútbol. RICYDE. Revista Internacional de Ciencias del Deporte, 11(42), 329-338.

\section{Dirección para correspondencia}

Luis Benavides Roca

Profesor de Educación Física.

Magíster en Ciencias de la Actividad Física, Universidad Católica del Maule, Chile.

Master Ciencias del deporte

U. Castilla la Mancha, España.

Grupo de investigación Muévete Chile.

Contacto:

benavides.roca@gmail.com

Recibido: 18/02/2018

Aceptado: 02/06/2018 